\title{
Experimental Assessment of Hydrocyclones with two Inlet (Feed) Gates
}

\author{
https://doi.org/10.3991/ijes.v6i3.9409 \\ Ebrahim Alamatian $^{(凶)}$, Ayda Nasiri-Kalat \\ Khavaran Institute of Higher Education, Mashhad, Iran \\ e.alamatian@profs.khi.ac.ir
}

\begin{abstract}
Hydrocyclone is a device to separate sediments in the water Gravel and sand particles are separated based on the specific weight of the suspended particles in the water by the ratio of their centripetal force to fluid resistance. Generally, hydrocyclone includes inlet (feed) and outlet flow gates. In this paper, one of the hydrocyclone, which is in the Wastewater Treatment Plant of Mashhad, has been investigated with respect to rising of the number of inlet flow gates. In other words, water enters to hydrocyclone with two inlets, which have made by welding and cutting operations. Results of this research show that the device efficiency has been enhanced 77 percent in sediment removal and the shear limit has been decreased 15 percent by increasing the number of the inlet gates. Assessments indicate the local temperature is not effective in the rate of particle removal in hydrocyclone.
\end{abstract}

Keywords-Hydrocyclone, Shear limit, Inlet gate, Efficiency, Pressure drop

\section{Introduction}

The existence of particles such as sand in drinking water has caused consumer's dissatisfaction, water pollution and an increase in chlorine use. Moreover, the pumps and water network systems have been eroded and the washing cost of the reservoir and water network supply have to be increased by these particles. Entering the gravel and particles into the water network supply declines water quality and settles into pipes, then the pipe would be destroyed and the pipe diameter would be increased as well. Furthermore, the valves and consumption measurement tools have damaged due to the particles. One of the straightforward and essential tools, in order to access to desirable water quality, is hydrocyclone in wells output and water network supply entrance due to high efficiency and low maintenance expenditures.

According to Figure 1, hydrocyclone consists of the main cylinder body, which has a central pipe named vortex finder conduct. The underside of the cylinder connects to a cone that ends part of it is called a spigot. In addition, an inlet pipe (feed) connects to the top part of the cylinder. Entry of tangential flow from the pipe causes rotation and centrifugal force. Primary and secondary vortexes are created by flow rotation and centrifugal force. Primary vortex takes roughing and suspended particles to the hydrocyclone wall and then moves towards spigot. Afterward, the second vortex leads to light 
and small particles accompanied with water through vortex finder conduct to the outlet gate.

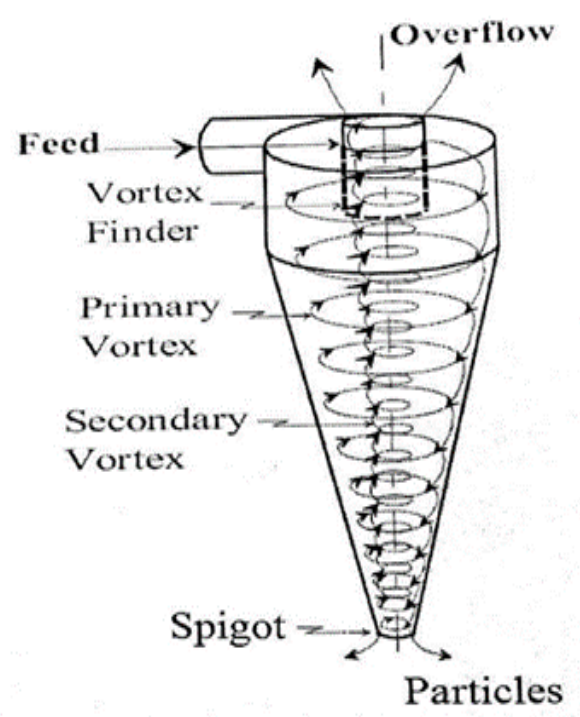

Fig. 1. A type of hydrocyclone

First research about hydrocyclone was done by Kelsall, [1]. They studied flow pattern details in hydrocyclone in experimentally way. He investigated small hydrocyclone by injecting the thin mixture of aluminum small particle and the movement observation by microscope. Kelsall measured axial and tangential velocity in a specified position and Calculated radius velocity by the continuity equations.

Monredon, et al., measured velocity profiles in 75 and $100 \mathrm{~mm}$ diameter of hydrocyclones by using laser velocity measurement, [2]. In this study, the effect of various variables such as vortex finder conduct diameter, spigot diameter and cone angle on velocity profiles were assessed. The results showed that the position of zero axial velocity did not change in the cylinder part by increasing the diameter of vortex finder conduct and spigot whereas it transferred towards inside in the cone part.

Narasimha et al. use the CFD model for simulation of the hydraulic parameter in hydrocyclone with water, air and sand particles, [3]. The performance of hydrocyclones was evaluated to determine their performance in the circuits and the current operational practices by Palaniandy et al., [4]. Hydrocyclone performance has been evaluated in one and two inlet gates by using numerical simulation, $[5,6,7,8]$. The results of the studies and comprising of two types of hydrocyclone indicates that flow parameters are close with together, while the distribution of them is different. Furthermore, flow symmetry has considerably enhanced. Velocity vector and velocity curves in the specified cross-section show asymmetry in the flow in the inlet cross-section decrease via getting away from the inlet. Moreover, efficiency measurement results indicate that efficiency 
has enhanced in the hydraulic cyclone with two gates. They described the reason of efficiency enhancement through using velocity vectors in the two-type hydrocyclone. According to his investigation in one gate-hydraulic cyclone, the major rate of flow moves towards the outlet gate and residual rate moves downward and towards sides. Therefore, the primary part leaves hydrocyclone without any suspended particles separation.

There are a few numbers of studies about two inlet gates, especially experimental methods, in hydrocyclones. On the other hand, hydrocyclone removal efficiency depends on sand properties in water. In this paper, created changes in sand and gravel Removal efficiency and shear limit are assessed by making two inlet flow gates in a hydraulic cyclone, which is installed in one the water, and wastewater organization wells.

\section{Materials and methods}

The main aim of this paper is that effect of the change in the number of flow inlet gates into hydrocyclone experimentally is assessed. Regarding this aim, it is selected an active well equipped with the hydraulic cyclone in Ghasem-Abad zone, Mashhad in Iran. The well is active diurnally in 24 hours and it enters 90 cubics per second discharge to the distribution network directly.

In this study, the sand in the hydrocyclone discharges completely. These sediments have settled due to previous good operation. The discharged sediments show in Figure 2. Then, the hydrocyclone has been operated in the system again and the amount of sand, which had been separated from water by using hydrocyclone, was measured in eight days. This process was performed by collecting sediments in 200-liter reservoir and testing them in the soli mechanic laboratory. Grading, weight and sediments shear limit was determined in the lab. The examination was done two times. Then, change in the flow inlet into hydrocyclone was implemented by welding and cutting operations. By applying the alterations, the flow enters to hydrocyclone through two inlet gates. The changes in hydrocyclone are shown in Figure 3 to 5.

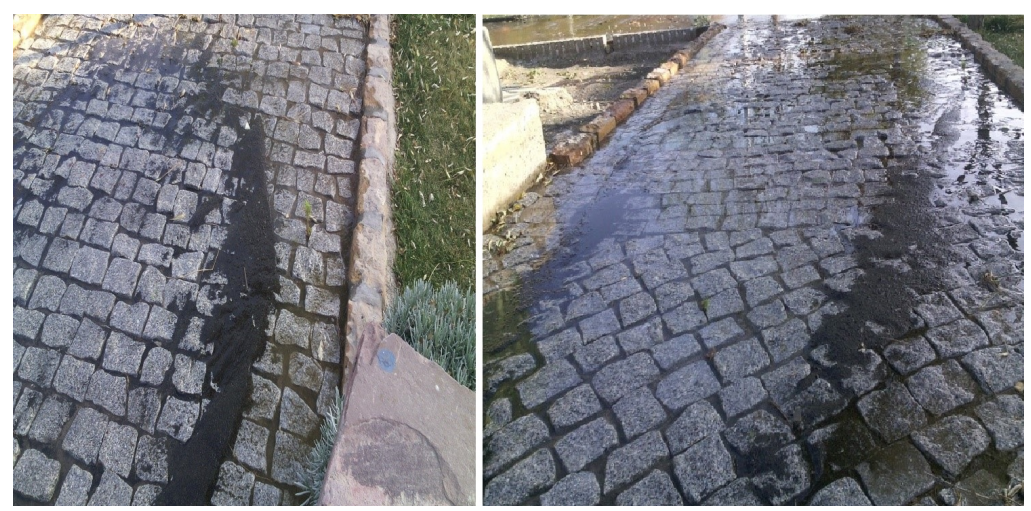

Fig. 2. Discharged sand from hydrocyclone before conducting tests 
Inlet pipe, which has $150 \mathrm{~mm}$ in diameter, is cut and changed by two pipes of $80 \mathrm{~mm}$ in diameter in hydrocyclone instead. Applying two pipes of $80 \mathrm{~mm}$ in diameter is due to minimizing flow inlet cross-section to hydrocyclone. In other words, this change is happened because of maintaining constant inlet velocity to hydrocyclone. It is tried to maximize tangential velocity via variations on flow inlet gate geometry. In addition, inlets make in the same axis in order to prevent flow inlet turbulence to hydrocyclone. When physical changes had been conducted, hydrocyclone was taken into the system and washed. Then it is tested two times again in the period ( 8 days). Eventually, the obtained samples were tested.
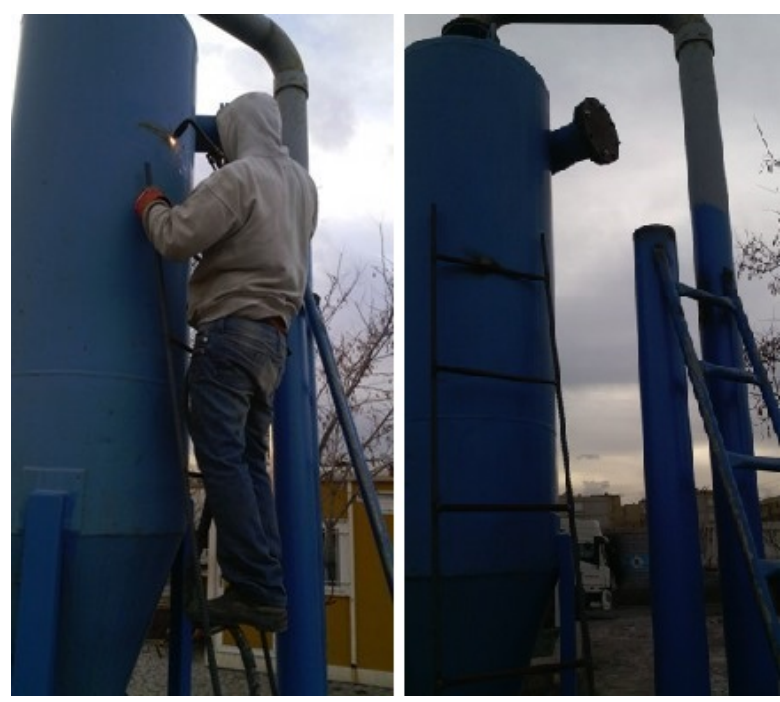

Fig. 3. Stages of cutting inlet gate to hydrocyclone

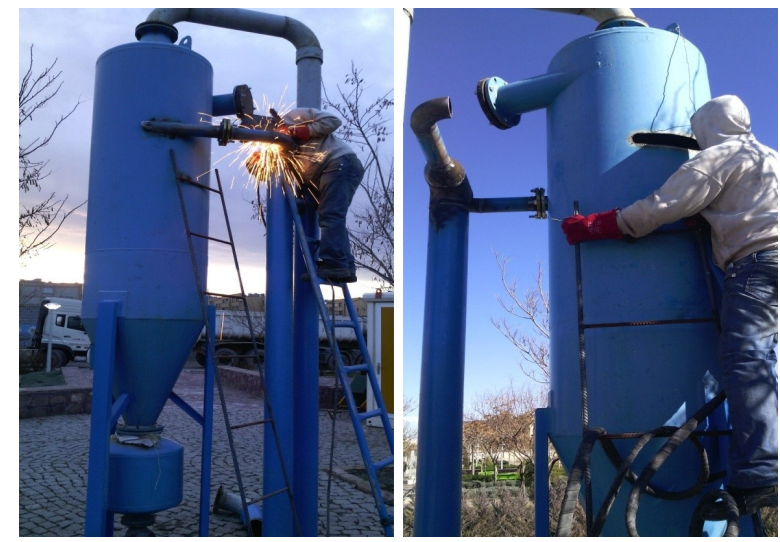

Fig. 4. Stages of cutting inlet gate to hydrocyclone 

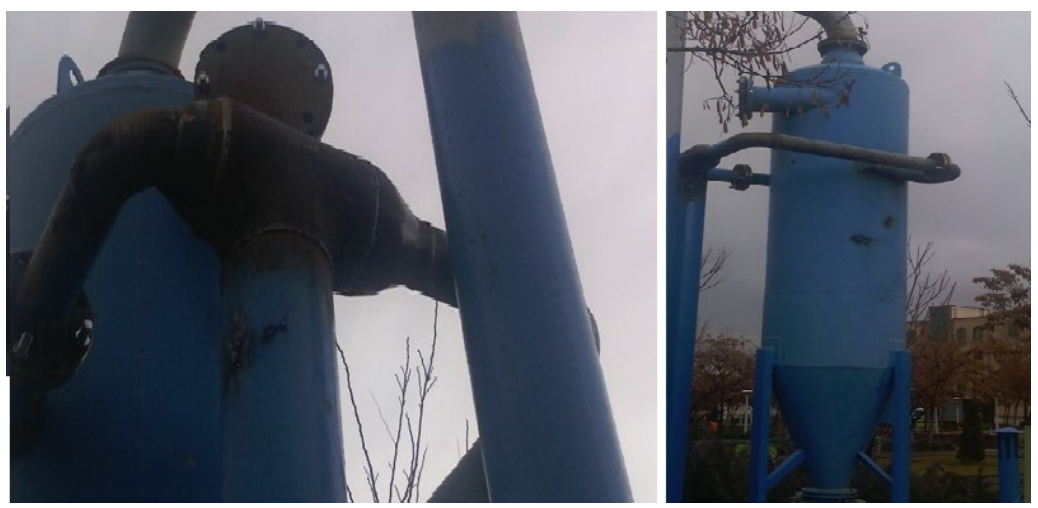

Fig. 5. Completion of the installation operation and change in the inlet gate

\section{Grading results}

According to ASTM classification, gravel particles refers to aggregates that are smaller than $75 \mathrm{~mm}$ and bigger than $4.75 \mathrm{~mm}$. Aggregates which are between 4.75 and $0.75 \mathrm{~mm}$, are called sand. Moreover, particles which are smaller than $0.075 \mathrm{~mm}$ are named silt and clay. In this research, particles are called sand in regard to aggregates are smaller than $4.75 \mathrm{~mm}$ in the current studies.

It is applied sieve analysis or gradation test to classify sand particles and sieves with the specific opening are used. Therefore, sieves, which have bigger and smaller openings, place in the top to down, respectively. The sieves are shacked in Specified time in order to passing sand and gravel particles according to ASTM standard. Obviously, particles, which are smaller than sieves opening pass and bigger ones, remain on the sieves.

Gradation results of sand in hydrocyclone reservoir with one inlet gate are shown in Figure 6. The amount of collected Sand in hydrocyclone reservoir with one inlet gate during the test was $353.1 \mathrm{gr}$. The size of the particles is between 75 and 3000 microns in the hydrocyclone. It shows that there are not bigger particles than 3000 micron in outlet water from well due to existence of the meshes, which have $3 \mathrm{~mm}$ diameter, in the pipes of the good side. The limitation of the mesh diameter stops entering particles, which are larger. Moreover, particles, which are smaller than 75 microns, was not collected by the hydraulic cyclone. It indicates that smaller particles than former diameter did not enter the well or the hydrocyclone could not remove them. It considers that maximum particles belong to the No. 30 sieve, which its opening is 600 micron and minimum particles refer to the No. 200 sieve, which its opening is 75 micron. 


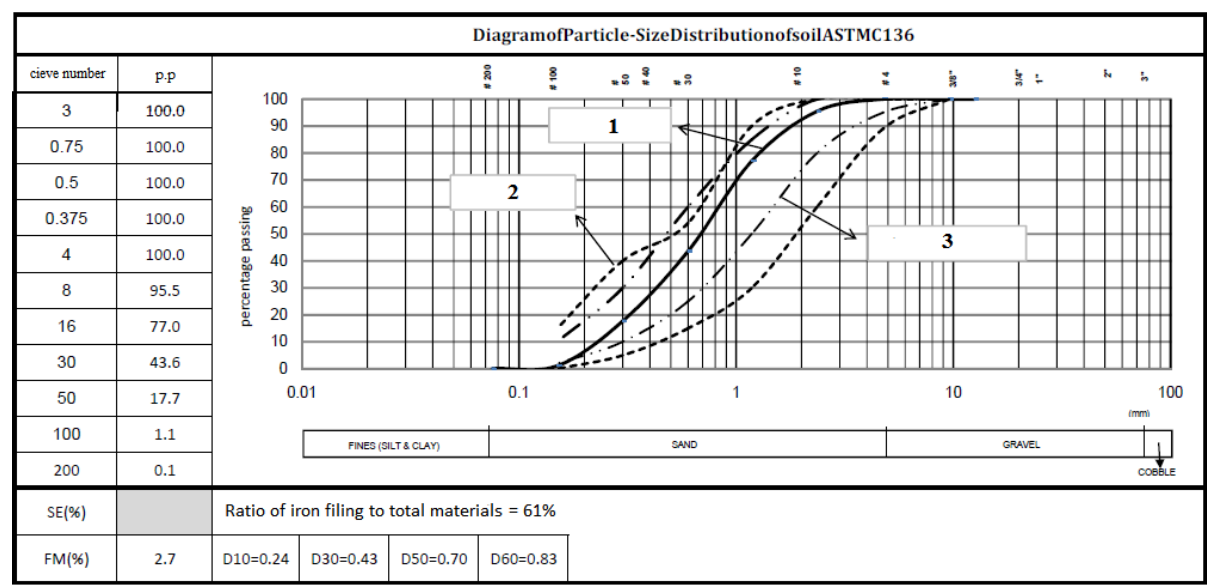

Fig. 6. Hydrocyclone grading curve with one inlet gate (1: Grading curve, 2: Grading envelope according to Building Regulations of Iran [9], 3: Grading envelope according to Iranian Road Construction [10])

Gradation results of sand in hydrocyclone reservoir with two inlet gates are shown in Figure 7. The amount of separated Sand in hydrocyclone reservoir with two inlet gates during the test period is $625.2 \mathrm{gr}$. The maximum and minimum sizes of the collected particles are 2360 and 75 micron, respectively. The plentitude of the particles based on weight measurement is $8.19 \mathrm{gr}$ in the No. 200 sieve ( 75 microns). The result compared to the results of hydrocyclone with one inlet gate, increases 55 percent in sediments collection. Moreover, the amount of the collected sand on the No. 100 sieve (150 microns) is $130.22 \mathrm{gr}$. The result indicates that the amount of collected sand compared to it in hydrocyclone with one inlet gate rises 55 percent. Also, collected sand has increased 55, 45, 43 and 30 percent on sieves of the No. 100 (150 microns), the No. 50 (300 microns), the No. 30 (600 microns) and the No. 16 (1180 micron), respectively. On the other, the results show that collected sand on the No. 8 sieve (2360 micron) Decreases 8 percent. Furthermore, it is observed that the amount of collected sand in hydrocyclone with two inlet gates has risen 77 percent which illustrates the Superior performance of hydrocyclone with two inlet gates compared to one inlet gate.

The percentage of the sand removal is higher when the sizes of particles are smaller and also shear limit decreases $0.7 \mathrm{~mm}$ to $0.6 \mathrm{~mm}$ in hydrocyclone with two inlet gates. Investigating this function depicted that hydrocyclone with two inlet gates can remove sands particles with smaller sizes and also, decrease shear limit of about 15 percent. Decreasing in shear limit and increasing in separated sand present better efficiency in hydrocyclone with two inlet gates compared to one inlet gate. 


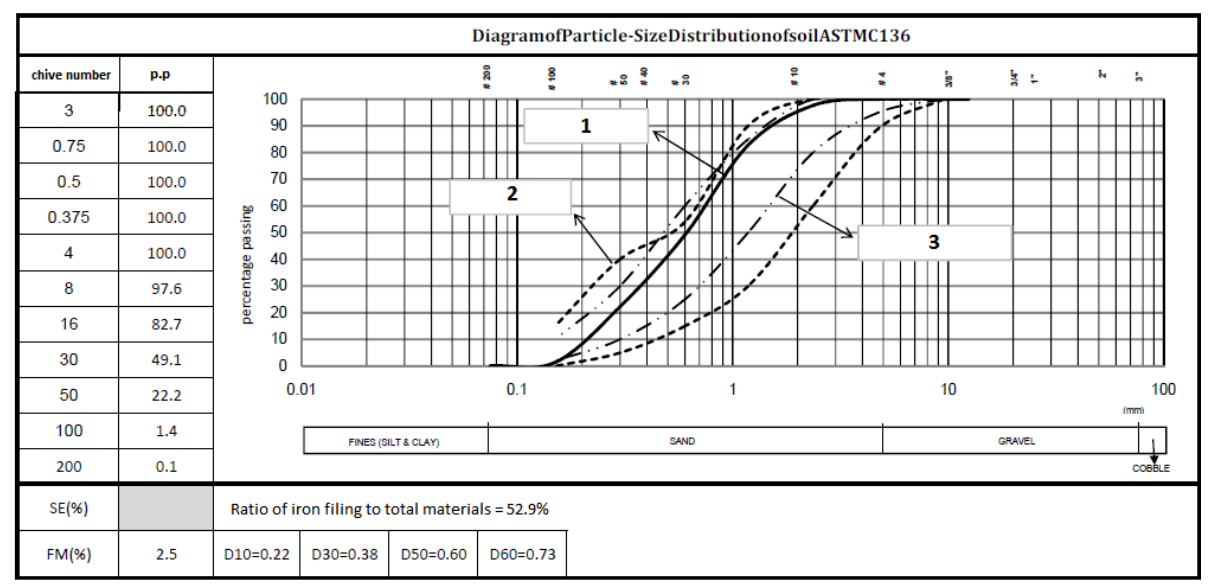

Fig. 7. Hydrocyclone Grading curve with two inlet gates (1: Grading curve, 2: Grading envelope according to Building Regulations of Iran [9], 3: Grading envelope according to Iranian Road Construction [10])

Studies show that a large number of iron filings accompany with collected sands. Iron filings have created due to crashing sand to pump and pipe sides and taking off them. It shows that $215 \mathrm{gr}$ of the $353.1 \mathrm{gr}$ collected sand which is equal to 61 Percent of total particles, is iron filings that have been separated by the magnet; therefore, the rest of them is collected sand particles sands particles. Also, $345 \mathrm{gr}$ of the $625.2 \mathrm{gr}$ collected sand refer to iron filings which are 52.9 Percent of total particles and the rest of them are sand particles. These results illustrate that the amount of iron filings that have been removed by hydrocyclone with two inlet gates is more compared to one inlet gate according to weight analysis. However, the removal percent of iron filings in hydrocyclone with two inlet gates is less compared to one inlet gate. Regarding similar velocity and discharge and the same amount of iron filings that separate from pump and pipe well sides, these results demonstrate higher efficiency of hydrocyclone with two inlet gates compared to one inlet gate.

It is shown the amount of separated sand and Accumulation percentage in Hydrocyclones with one and two inlet gates in Figure 8 and 9, respectively. As it can be seen in Figure 8, the amount of collected sand in any sieve is more in hydrocyclone with two inlet gates compared to one inlet gate. Moreover, the maximum amount of Separated sand particles finds in the No. 30 sieve and the No. 50 in both hydrocyclone with one and two inlet gates. The behavior indicates that the majority of particles are in the sieves number limit. According to the curves, minimum particles removal refers to the No. 8 and No. 200 sieves. Since roughing particles (almost the No. 8 sieve) separate hardly from the good side due to the existence of well side pipe meshes. Furthermore, a low amount of separated particles around the No. 200 sieve is owing to low hydrocyclone performance. Also, it is observed that particle size Amplitude is constant and only, the amount of separated sand is variable by each sieve. 


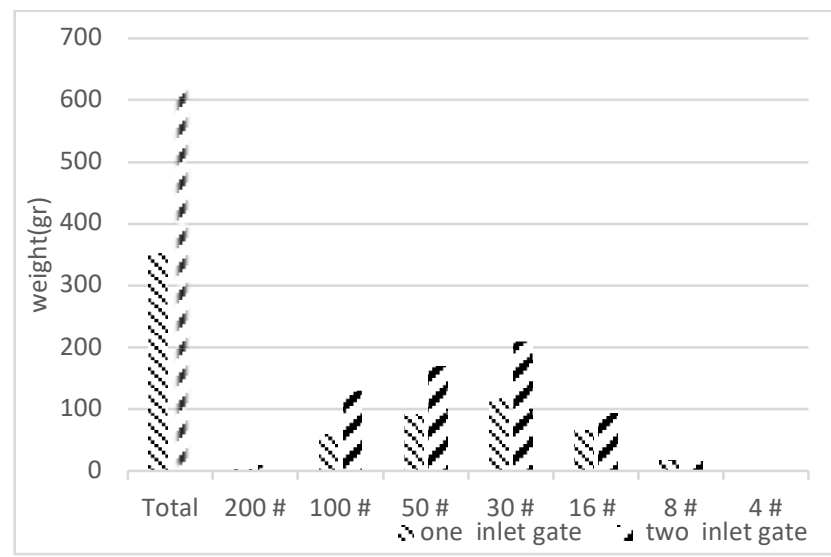

Fig. 8. The amount of separated sand in hydrocyclone with one and two inlet gates

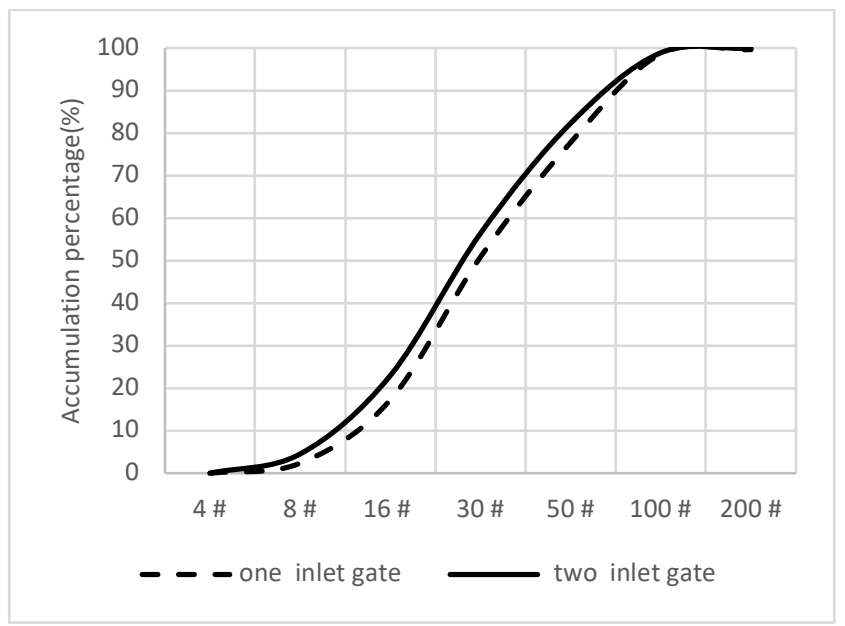

Fig. 9. Comparing Collected particles percentage in hydrocyclone with one and two inlet gates

\section{Conclusion}

In this paper, it was studied the effect of the change in inlet gate (feed) on a hydrocyclone, which is installed in a well in Mashhad in Iran. The results show that current hydrocyclone does not have desirable performance. Especially for small particles. The results of the study indicate that particle removal efficiency has increased 77 percent and also shear limit decreased 15 percent as the one inlet gate changed to two inlet gates without changing in size and inlet flow parameters values which causes symmetry flow entering to hydrocyclone. Assessing of collected sand weight in hydrocyclone with two inlet gates depict 100 percent increment in the sand collection from the well. Furthermore, results show that a considerable amount of iron filings accompany with hydrocyclone sediments. 


\section{Acknowledgment}

The support of the research council of Mashhad Water and Wastewater Company during the preparation of this work is kindly acknowledged.

\section{References}

[1] Fisman, L. (2005). The effects of local learning on environmental awareness in children: An empirical investigation. The Journal of Environmental Education, 36(3), 39-50. https://doi.org/10.3200/JOEE.36.3.39-50

[2] Kelsall, D. F. "A further study of the hydraulic cyclone." Chemical Engineering Science. 2.6(1953), 254-272. https://doi.org/10.1016/0009-2509(53)80044-8

[3] Monredon, T. C., K. T. Hsieh, and Raj K. Rajamani. "Fluid flow model of the hydrocyclone: an investigation of device dimensions." International Journal of Mineral Processing. 35.1(1992), 65-83. https://doi.org/10.1016/0301-7516(92)90005-H

[4] Narasimha, M. Brennan, M.S and Holtham, P.N. "CFD modeling of hydrocyclones: Prediction of particle size segregation." Minerals Engineering, 39(2012), 173-183. https://doi.org/10.1016/j.mineng.2012.05.010

[5] Palaniandy, S., Yahyaei, M. and Powell, M. "Assessment of hydrocyclone operation in gravity induced stirred mill circuits" Minerals Engineering, 108(2017), 83-92. https://doi.org/10.1016/j.mineng.2017.01.002

[6] Yoshida H, Yoshikawa S, Fukui K, Yamamoto T. "Effect of multi-inlet flow on particle classification performance of hydrocyclones." Powder Technology. 184.3(2008), 352-360. https://doi.org/10.1016/j.powtec.2007.09.008

[7] Safikhani, H., Akhavan-Behabadi, M.A., Shams, M., Rahimyan, M.H., "Numerical simulation of flow field in three types of standard cyclone separation." Advanced powder Technology, 21(2010), 435-442.

[8] Medronoho, R.A., schuetze, J., Deckwer, W.D., "Numerical simulation of hydrocyclones for cell separation." Latin American applied research, 35(2005), 1-8.

[9] Salehi Far, E, Moghiman, M, "Numerical simulation and investigation of the effect of double inlets on hydrocyclone performance." Mining Engineering conference, 9(2013).

[10] National Building Regulations of Iran. 2013 Design and implementation of reinforced concrete buildings, Issue 9, Ministry of Roads and City Planning, Tehran, Iran.

[11] Formulation of Technical Criteria of Iran. 2003 General technical specifications of the road, Bulletin number 101, Management and planning organization, Tehran, Iran.

\section{$7 \quad$ Authors}

Ebrahim Alamatian is an assistant professor in Khavaran Institute of Higher Education, Baradaran Shahid Hosseini 12th Alley, Fallahi Blvd, West Town, Mashhad, Iran. (E.Alamatian@profs.khi.ac.ir)

Ayda Nasiri-Kalat received the Master degree in Civil Engineering-Environment from the Khavaran Institute of Higher Education. (ayda.nasiri1992@gmail.com)

Article submitted 21 August 2018. Final acceptance 06 October 2018. Final version published as submitted by the authors. 